metrics of night work (e.g. duration, lifetime mean no. nights per month and cumulative no. nights) and risk of breast cancer, and whether the association was dependent on age or change in body weight.

Methods Overall, 55,350 female nurses completed extensive questions on shift work and were followed for incident breast cancer. Cox regression yielded multivariable-adjusted breast cancer incidence hazard ratios (HRs) and 95\% confidence intervals (CIs) for night work groups vs never night work, and Wald tests were used to assess potential interaction with age and change in body weight.

Results During 5,5 years of follow-up, 687 cases of incident invasive breast cancer were registered. The age adjusted HRs were 1.26 (95\% CI 1.01-1.57) for ever vs never night work, and 1.32 (95\% CI 1.01-1.74) for the tertile with the greatest cumulative no. nights ( $\geq 991$ nights) vs never night work. No trends were observed with increasing levels of different exposure metrics. There was a statistically significant interaction between night work and weight gain on risk of breast cancer; among women with $12-18 \mathrm{~kg}$ weight gain since age 18 , the HR for ever vs never night work was 2.45 (95\% CI 1.28 to 4.68).

Conclusion Higher duration, mean no. nights per month and cumulative no. nights were associated with a moderately increased risk of invasive breast cancer. Particularly for night shift workers with weight gain of $12-18 \mathrm{~kg}$, the risk was increased. Our results point towards a potential interaction between change in body weight and night shift work on breast cancer risk.

\section{Specific Occupations}

\section{0-57 ALUMINUM DUST EXPOSURE AND RISK OF NEURODEGENERATIVE DISEASES IN A COHORT OF MALE MINERS IN ONTARIO, CANADA}

'Xiaoke Zeng, Jill MacLeod, Colin Berriault, Nathan DeBono, Victoria H Arrandale, Anne M Harris, Paul Demers. 'University of Toronto, Canada

\subsection{6/OEM-2021-EPI.144}

Introduction McIntyre Powder (MP), a fine-sized aluminum and aluminum compound powder, was administered to Ontario miners from 1943 to 1979 as purported prophylaxis against silicosis. Aluminum has long been suspected of having a role in the development of neurological diseases. However, very few studies have examined the risk of neurological disease among miners with exposure to aluminum dust, and previous findings were inconclusive.

Objectives We estimated associations between respirable aluminum exposure through MP and neurological disease in a retrospective cohort of mining workers from Ontario, Canada. Outcomes included Alzheimer's disease, Alzheimer's with other dementias, Parkinson's disease, parkinsonism, and motor neuron disease.

Methods The cohort was created by linking a database of mining workers' work history to health care records. This analysis included 36,826 male miners potentially exposed to MP between 1943 and 1979, followed up for disease diagnosis between 1992 and 2018. Exposure was assessed using two approaches, self-reported and historical records. Neurological diseases were ascertained using physician billing and hospital discharge records. Poisson regression models were used to estimate associations between MP exposure and neurological outcomes using incidence rate ratios and 95\% confidence intervals (RR, 95\% CI).

Results Exposure to self-reported MP was associated with an elevated incidence rate of Parkinson's disease (RR 1.34, 95\% CI: 1.14, 1.57). The rate of Parkinson's disease appeared to increase with the duration of exposure assessed by historical records. Ever-exposure to MP was positively associated with an elevated rate of Alzheimer's with other dementias (RR 1.12, 95\% CI 1.06, 1.19), but not $\mathrm{Alz}$ heimer's disease alone.

Conclusion This study found that miners who were exposed to respirable aluminum, as McIntyre Powder, had elevated rates of Parkinson's disease. The rate of Parkinson's disease appeared to increase with the duration of exposure assessed by historical records.

\section{0-105 MATERNAL OCCUPATION AS A NAIL TECHNICIAN OR HAIRDRESSER DURING PREGNANCY AND BIRTH DEFECTS, NATIONAL BIRTH DEFECTS PREVENTION STUDY, 1997-2011}

${ }^{1}$ Miriam R Siegel, Carissa M Rocheleau, Kendra Broadwater, Albeliz Santiago-Colón, Michele L Herdt, I-Chen Chen, Christina C Lawson, Candice Johnson. ${ }^{1}$ CDC/NIOSH, United States

\subsection{6/OEM-2021-EPI.145}

Introduction Nail technicians and hairdressers may be exposed to products containing chemicals with potential reproductive effects. While studies have examined birth defects in children of cosmetologists and hairdressers, nail technician work has not been individually evaluated as a risk factor for birth defects.

Objectives We investigated associations between maternal occupation as a nail technician or hairdresser during pregnancy (versus non-cosmetologist) and selected birth defects.

Methods We analyzed population-based case-control data from the multisite National Birth Defects Prevention Study, 19972011. Cases were fetuses or infants with major structural birth defects; controls were liveborn infants without major birth defects. For 31,652 case and 11,613 control mothers, expert raters classified self-reported maternal jobs into discrete categories as nail technician, hairdresser, combination nail technician-hairdresser, other cosmetologist, or non-cosmetologist. We used logistic regression to calculate odds ratios (ORs) and 95\% confidence intervals (CIs) for associations between occupation during the first trimester of pregnancy and birth defect type, controlling for age, smoking, education, and race/ ethnicity.

Results Sixty-one mothers worked as nail technicians, 196 as hairdressers, 39 as combination nail technician-hairdressers, and 42,810 as non-cosmetologists during pregnancy. Strongest associations among nail technicians included multiple congenital heart defect (CHD) groups: any $\mathrm{CHD}(\mathrm{OR}=2.7$; $\mathrm{CI}: 1.3-$ 5.9); conotruncal $(\mathrm{OR}=3.0$; $\mathrm{CI}: 1.0-8.8)$ (Tetralogy of Fallot $[\mathrm{OR}=3.5 ; \mathrm{CI}: 1.0-12.9])$; right ventricular outflow tract obstruction $(\mathrm{OR}=3.2$; CI: $1.0-10.4)$; and septal $(\mathrm{OR}=3.1$; CI: 1.2-8.1). Cleft lip with cleft palate was associated with occupation as a hairdresser $(\mathrm{OR}=2.0 ; \mathrm{CI}$ : 1.1-3.7). All oral cleft groups were associated with combination nail technician-hairdresser work (ORs ranging from 4.2 to 5.3).

Conclusion Despite small samples, results suggest associations between maternal nail technician work during pregnancy and 
various CHDs and similarly between hairdresser work and oral clefts. Future research could aim to identify potential workplace reproductive hazards and interventions in these occupations.

\section{0-147 SENSITIZATION AND DERMATITIS AMONG EPOXY EXPOSED LAMINATION WORKERS PRODUCING WIND TURBINE BLADES}

${ }^{1}$ Alexandra Golabek Christiansen, Ole Carstensen, Henrik Albert Kolstad, Jakob Hjort Bønløkke, Per Axel Clausen, Marléne Isaksson, Mette Sommerlund, Vivi Schlünssen. ${ }^{1}$ Aarhus University Hospital, Denmark

10.1136/OEM-2021-EPI.146

Introduction Epoxy resin systems (ERS) are well-known sensitizers of the skin. A high prevalence of sensitization and dermatitis has been reported among workers exposed to ERS. Due to this, comprehensive personal protective equipment is required when working with ERS. No recent studies have evaluated the effect of the use of such safety equipment.

Objectives The aim of this study was to estimate the occurrence of dermatitis and sensitization to ERS among epoxyexposed workers producing wind turbine blades in Denmark while using up-to-date protective measures.

Material and Methods A cross-sectional study was performed at two Danish factories producing rotor blades for wind turbines. A screening questionnaire regarding recent and former skin rashes, allergies, atopic dermatitis, and asthma was answered by 181 epoxy-exposed production workers and 41 non-exposed office workers. Physical examination of the skin was followed by testing with a tailored patch test series based on toxicological assessments of possible sensitizing chemicals in the work materials including epoxy resins and hardeners as well as 35 allergens from the European Standard Series (TRUE test). Atopy was defined as elevated serum levels of $\operatorname{IgE}$ for standard inhalation allergens.

Results In total, 16 (8.8\%) of the exposed workers were sensitized to one or more epoxy compounds, whereas none of the non-exposed office workers were sensitized. Non-atopic participants exposed to epoxy products showed an increased odds ratio $(\mathrm{OR}=2.02$; CI $0.56-7.34)$ of dermatitis while the opposite was seen for atopic participants $(\mathrm{OR}=0.08$; CI $0.02-0.4)$. A 4-fold increased odds ratio $(\mathrm{OR}=4.5$; CI 1.57-13.13) of dermatitis was observed among workers sensitized to epoxy resins. Atopy was not associated with epoxy sensitization $(\mathrm{OR}=0.73$, CI $0.22-2.42)$.

Conclusion Despite up-to date skin protection dermatitis and sensitization to ERS remain high among epoxy exposed lamination workers. These findings document the need for new and efficient preventive efforts.

\section{0-217 ASBEST CHRYSOTILE COHORT STUDY PROFILE, EXPOSURE DISTRIBUTION AND OUTCOMES}

${ }^{1}$ Ann Olsson, Joachim Schuz, Kurt Straif, Hans Kromhout, Monika Moissonnier, Evgenia Ostroumova, Graham Byrnes, Gilles Ferro, Eleonora Feletto, Sara J Schonfeld, Igor Bukhtiyarov, Iraklii Tskhomariia, Sergey Kashanskiy, Tatiana Morozova, Evgeny Kovalevskiy. 'International Agency for Research on Cancer, France

\subsection{6/OEM-2021-EPI.147}

Introduction A historical cohort study in workers occupationally exposed to chrysotile was set up in the town of Asbest, the Russian Federation, to study their cause-specific mortality, with a focus on cancer.

Objective Describe the Asbest Chrysotile Cohort established in 2013.

Methods Cohort enrolment was based on employment records from JSC Uralasbest chrysotile mine and processing factories. Exposure assessment was based on detailed occupational histories extracted from company archives and personal workbooks linked to $>90,000$ measurements, and conversion factors derived using a subset of parallel dust/fibre measurements. This resulted in yearly dust and fibre estimates for each employed calendar year. Vital status was ascertained from multiple sources including company records, the Pension Fund, the Federal Migration Service, and Civil Act Registration Office providing the date and cause of death of those deceased in Sverdlovsk Region.

Results The cohort comprises 22,463 men and 13,374 women working $\geq 1$ year between $01 / 01 / 1975$ and $31 / 12 / 2010$ in JSC Uralasbest and followed until 31/12/2015. Cumulative exposure of exposed women was higher than for men $(49 \mathrm{mg} / \mathrm{m} 3$ dust years and 33 fibres $/ \mathrm{cm} 3$ years vs. $31 \mathrm{mg} / \mathrm{m} 3$ dust years and 19 fibres/cm3years). At the end of follow-up, 52\% of the cohort was alive, $36 \%$ deceased, and 12\% censored at last date known to be alive in the Sverdlovsk Region. For those who died the mean age at death was 59.4 years for men and 66.5 for women. The most frequent underlying cause of death for all was circulatory diseases, followed by external causes and cancer in men, and cancer and external causes in women. Cancer mortality was dominated by lung cancer in men and breast cancer in women.

Conclusion The cohort is unique in its size, long follow-up, and substantial proportion of female workers. The cohort is relatively young, and the mortality patterns follow those of the Russian population. Risk analyses are underway.

\section{0-231 HEALTH, LIFESTYLE AND OCCUPATIONAL RISKS IN 10,931 INFORMATION TECHNOLOGY WORKERS}

Jim Lewsey, Srinivasa Vittal Katikireddi, Ewan Macdonald, Evangelia Demou, ${ }^{1}$ Drushca Lalloo. 'University of Glasgow, United Kingdom

\subsection{6/OEM-2021-EPI.148}

Introduction Informational technology (IT) and the IT workforce are rapidly expanding with potential occupational health implications. Yet to date, IT worker health is under-studied and large-scale studies are lacking.

Objectives To investigate health, lifestyle and occupational risk factors of IT workers.

Methods We evaluated self-reported health, lifestyle and occupational risk factors for IT workers in the UK Biobank database. Using logistic regression, we investigated differences between IT workers and all other employed participants. Regression models were repeated for IT worker sub-groups (managers, professionals, technicians) and their respective counterparts within the same Standard Occupational Classification (SOC) major group (functional managers, science and technology professionals, science and technology associate professionals).

Results Overall, 10,931 (4\%) employed participants were IT workers. Compared to all other employed participants, IT workers reported similar overall health, but lower lifestyle risk factors for smoking and obesity. Sedentary work was a 